\title{
Optical Identifications of Five INTEGRAL Hard X-ray Sources in the Galactic Plane
}

\author{
I. F. Bikmaev ${ }^{1,2 *}$, R. A. Burenin ${ }^{3}$, M. G. Revnivtsev ${ }^{3,4}$, S. Yu. Sazonov ${ }^{3,4}$, \\ R. A. Sunyaev ${ }^{3,4}$, M. N. Pavlinsky ${ }^{3}$, and N. A. Sakhibullin ${ }^{1,2}$ \\ ${ }^{1}$ Kazan State University, ul. Kremlevskaya 18, Kazan, Russia \\ ${ }^{2}$ Academy of Sciences of Tatarstan, ul. Baumana, 20, Kazan, Russia \\ ${ }^{3}$ Space Research Institute, ul. Profsoyuznaya 84/32, Moscow, 117997 Russia \\ ${ }^{4}$ Max-Planck Institut für Astrophysik, Karl-Schwarzschild-Str. 1, Garching, 85741 Germany \\ Received April 9, 2008
}

\begin{abstract}
The results of optical identifications of five hard X-ray sources in the Galactic plane from the INTEGRAL all-sky survey are presented. The X-ray data on one source (IGR J20216+4359) are published for the first time. The optical observations were performed with the 1.5-m RTT-150 telescope (Turkish National Observatory, Antalya, Turkey) and the 6-m BTA telescope (Special Astrophysical Observatory, Nizhny Arkhyz, Russia). A blazar, three Seyfert galaxies, and a high-mass X-ray binary are among the identified sources.

PACS numbers: 95.85. Kr; 95.85.Nv; 95.85.Pw; 98.52.Cf; 98.54.Cm; 98.62.Is; 98.62.Py; 98.62.Tc; 98.62.Ve; 98.70.Qy; 98.70.Rz

DOI: $10.1134 / \mathrm{S} 1063773708100010$
\end{abstract}

Key words: X-ray sources, gamma-ray sources, active galactic nuclei, X-ray binaries, optical observations.

\section{INTRODUCTION}

The INTEGRAL all-sky survey that has been carried out in the last few years (see, e.g., Krivonos et al. 2007) provides an opportunity to search for nearby active galactic nuclei (AGNs), accreting white dwarfs, high-mass and low-mass X-ray binaries, symbiotic stars, etc. The INTEGRAL operating energy range (17-60 keV) allows one to be virtually free from the selection effects related to the photoabsorption of $\mathrm{X}$-ray emission both near the observed $\mathrm{X}$-ray source and on the line of sight in the Galactic interstellar medium.

A considerable number of hitherto unknown hard $\mathrm{X}$-ray sources have been discovered during this survey. Our group performs optical identifications of such sources in the northern sky with the RussianTurkish 1.5-m RTT-150 telescope (Bikmaev et al. 2006a, 2006b; Burenin et al. 2008). In this paper, we present the results of our identifications of another set of sources from the INTEGRAL all-sky survey located near the Galactic plane.

${ }^{*}$ E-mail: ilfan.bikmaev@ksu.ru

\section{OBSERVATIONS}

As usual, we chose a number of northern-sky objects $\left(\delta>-30^{\circ}\right)$ for which accurate positions in the sky were known from observations with the $\mathrm{X}$-ray telescopes onboard the ROSAT, Chandra, and SWIFT observatories. The Chandra data for several sources were obtained at the request of our group (Sazonov et al. 2005, 2008). We retrieved all of the necessary additional publicly accessible X-ray data from the HEASARC archive. ${ }^{1}$

The optical observations of the sources were carried out with the RTT-150 telescope in the spring and summer of 2007 using two instruments of this telescope, the CCD photometer based on a thermoelectrically cooled Andor CCD array and the low- and medium-resolution TFOSC spectromete. $\mathrm{r}^{2}$ Grism no. 15, which provided the highest optical efficiency and the widest spectral range (3300-9000 $)$ ), was used for the spectrometry; the spectral resolution was $\approx 15 \AA$ (FWHM). In addition, we managed to observe some of the objects with the SCORPIO spectrometer of the 6-m BTA telescope (Afanasiev

\footnotetext{
${ }^{1}$ http://heasarc.nasa.gov/

${ }^{2}$ http://astroa.physics.metu.edu.tr/tug/tfosc.html
} 
List of identified sources

\begin{tabular}{c|cc|c|c|c}
\hline Name & \multicolumn{2}{|c|}{$\alpha, \delta(\mathrm{J} 2000)$} & $R_{\mathrm{c}}$ & $z$ & Type \\
\hline RX J0137.7 +5814 & 013750.45 & +591411.6 & 17.63 & $?$ & BL Lac \\
IGR J20216+4359 & 202149.04 & +440039.4 & 19.14 & 0.017 & Sy2 \\
IGR J21343+4738 & 213420.37 & +473800.4 & 13.79 & - & HMXB \\
IGR J23206 +6431 & 232036.58 & +643045.2 & 19.41 & 0.07173 & Sy1 \\
IGR J23523+5844 & 235222.00 & +584532.7 & 18.62 & 0.1620 & Sy2 \\
\hline
\end{tabular}

Note. Sy1 and Sy2 denotes type-1 and type-2 Seyfert galaxies; BL Lac denotes a BL Lac object; and HMXB stands for a high-mass $\mathrm{X}$-ray binary.

and Moiseev 2005) in the fall of 2007. The data were reduced using the standard $\mathrm{IRAF}^{3}$ and $\mathrm{DECH}^{4}$ software packages (Galazutdinov 1992).

\section{RESULTS OF OBSERVATIONS}

The list of sources studied and their classification are given in the table. The coordinates of the optical objects are given at epoch J2000, according to the astrometric solutions for direct RTT-150 images, which were obtained relative to the USNO-B1.0 Catalog (Monet et al. 2003). The magnitudes were measured from direct RTT-150 images. The photometric calibration was performed using the observations of standard stars from Landolt (1992). Below, the X-ray data and the results of optical observations for each source are discussed in more detail.

RX J0137.7+5814. The position of the X-ray source RX J0137.7+5814 from ROSAT data (Voges et al. 1999) and the corresponding hard X-ray source from INTEGRAL data (Krivonos et al. 2007) coincides, within the error limits, with the bright radio source $87 \mathrm{~GB} 013433.2+575900$ whose position is known with an accuracy of $\approx 6^{\prime \prime}-10^{\prime \prime}$. Only one star with an optical magnitude $R<18$ lies within a circle $10^{\prime \prime}$ in radius (Fig. 1a).

The spectrum of this source was taken with RTT-150 during several nights. In addition, the spectrum of this object was also taken with the 6m BTA telescope using the SCORPIO spectrometer (Afanasiev and Moiseev 2005). Because of poor weather conditions during these observations, we failed to perform measurements with the required signal-to-noise ratio. Nevertheless, we were able to

\footnotetext{
${ }^{3}$ http://iraf.noao.edu

${ }^{4}$ http://www.gazinur.com/Download.html
}

obtain a spectrum comparable in quality to the RTT150 spectra.

The spectrum of the optical object obtained by summing the RTT-150 and BTA spectra and corrected for the Galactic extinction $E(B-V)=0.85$ is shown in Fig. 1b. Telluric absorption bands (near 6900, 7200, and $7600 \AA$ ) and, probably, some unidentified absorption lines (e.g., the line at $4914 \AA$ ) are clearly seen in this spectrum. However, the spectrum exhibits no detectable stellar absorption or emission lines that are observed in AGN spectra. Together with the presence of an intense radio emission, this suggests that RX J0137.7+5814 is a blazar or, more precisely, a BL Lac object. Further, more sensitive optical observations of the source are needed to measure its redshift.

IGR J20216+4359. The hard X-ray source IGR J20216+4359 was discovered in an incomplete set of INTEGRAL observations of the Cygnus region (observations from January 14, 2004, to November 4, 2004; orbits 153-251). In this series of observations, the source was detected at a high confidence level $\left(\approx 5.5 \sigma\right.$; Fig. 2). Its coordinates are $\alpha=20^{\mathrm{h}} 21^{\mathrm{m}} .8$ and $\delta=+43^{\circ} 59^{\prime}$ (J2000) and its positional accuracy is $\approx 3^{\prime}$. The hard $\mathrm{X}$-ray $(17-60 \mathrm{keV})$ flux from the source was $\approx 1.1 \mathrm{mCrab}$, which corresponds to an energy flux of $\approx 1.6 \times 10^{-11} \mathrm{erg} \mathrm{s}^{-1} \mathrm{~cm}^{-2}$ for a powerlaw spectrum with a photon index of 2 . The source's flux is detected at a confidence level of only $\approx 3 \sigma$ in the complete set of observations of the Cygnus region, which corresponds to a flux of $0.6 \pm 0.2 \mathrm{mCrab}$.

The sky field around IGR J20216+4359 was observed by the ASCA observatory on June 10, 1993. The source was detected at a statistically significant level in these observations. This allowed its coordinates $\alpha$ and $\delta$ to be improved, $20^{\mathrm{h}} 21^{\mathrm{m}} 48^{\mathrm{s}} .1$ and $+44^{\circ} 00^{\prime} 32^{\prime \prime}$ (J2000, the accuracy is $\approx 20^{\prime \prime}$, Fig. 3), 
(a)
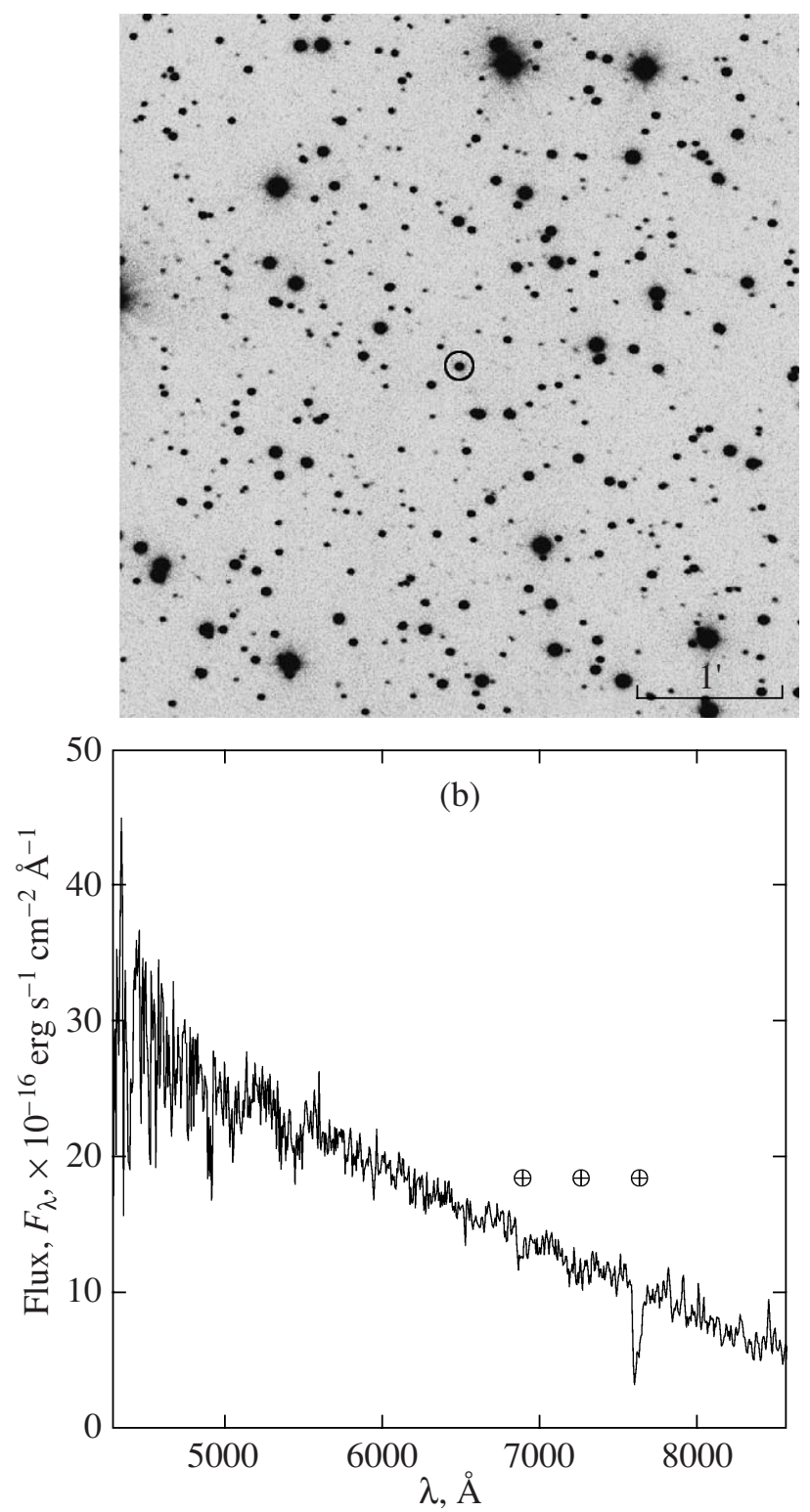

Fig. 1. (a) Optical image of the sky field around the source RX J0137.7+5814 from RTT-150 $R_{\mathrm{c}}$-band observations. The circle marks the error region of the radio source $87 \mathrm{~GB} 013433.2+575900$ in the sky in the shape of a circle with a radius of $\approx 6^{\prime \prime}$. (b) Combined optical spectrum of RX J0137.7+5814 obtained from RTT-150 and BTA observations and corrected for the Galactic extinction $E(B-V)=0.85$.

and the spectrum in the standard X-ray range $0.8-$ $10 \mathrm{keV}$ to be measured. The source turned out to be strongly absorbed, i.e., the equivalent photoabsorption column density measured from the shape of its X-ray spectrum is $N_{\mathrm{H}} L=(13 \pm 2) \times 10^{22} \mathrm{~cm}^{-2}$ (the photon spectral index was rigidly fixed at $\Gamma=1.7$ because of poor statistics). This value is considerably higher that the photoabsorption column density in the Galactic interstellar medium, $\approx 10^{22} \mathrm{~cm}^{-2}$ (Dickey and Lockman 1990). Such a strong internal X-ray photoabsorption is typical of type-2 Seyfert galaxies.

We discovered a Seyfert galaxy in the error box of IGR J20216+4359 using optical RTT-150 observations. This galaxy is marked by the circle in Fig. 3. Its spectrum exhibits forbidden [O III] and [N II] emission lines, which are indicative of a high AGN activity (see, e.g., Baldwin et al. 1981; Kauffmann et al. 2003). The absence of broad $\mathrm{H}_{\alpha}$ and $\mathrm{H}_{\beta}$ lines in the AGN 


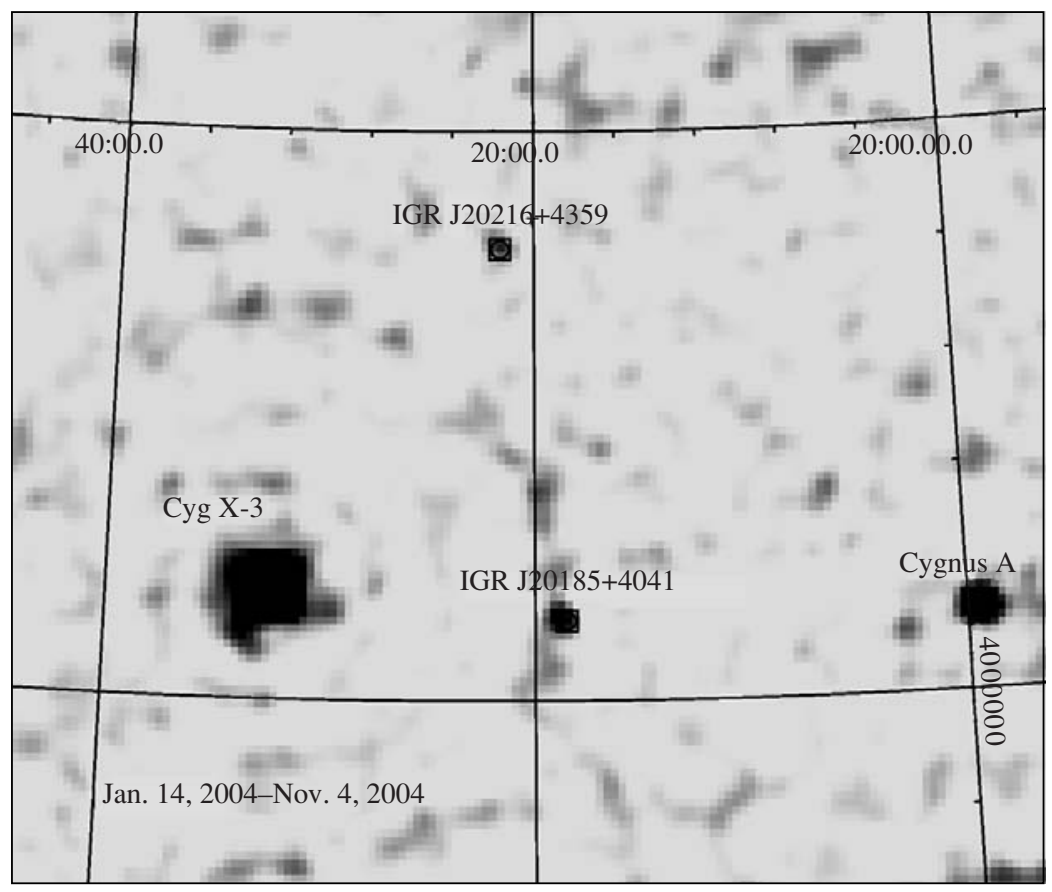

Fig. 2. IBIS/INTEGRAL image of the sky field around the source IGR J20216+4359 in the energy range 17-60 keV.

spectrum confirms the classification of this AGN as a type-2 Seyfert galaxy. The redshift of the galaxy was measured from narrow [O III] and [N II] lines, $z=0.017$.

IGR J21343+4738. The hard X-ray source IGR J21343+4738 was discovered during deep observations of the Galactic-plane region in Cygnus (Krivonos et al. 2007; Bird et al. 2007). More detailed studies of the source's behavior showed it to be variable. Figure 5 shows the IBIS/INTEGRAL images of the sky field around IGR J21343+4738 in different observing periods. IGR J21343+4738 is detected at a statistically significant level in the IBIS/INTEGRAL observational data only in the series of observations from December 27, 2002, to February 21, 2004 (Fig. 5a). The mean 17-60 keV flux from the source was $1.6 \pm 0.3 \mathrm{mCrab}$ in this series of observations, which corresponds to a flux of $\approx(2.3 \pm 0.4) \times 10^{-11} \mathrm{erg} \mathrm{s}^{-1} \mathrm{~cm}^{-2}$.

The source is not seen in the map of this sky field averaged over the period from March 11, 2004, to February 10, 2007 (Fig. 5b). In this series of observations, the exposure time was much longer than that in the first one and the upper limit on the source's $17-60 \mathrm{keV}$ flux was $0.5 \mathrm{mCrab}(2 \sigma)$, suggesting that it is transient in nature. Owing to the higher sensitivity, the AGN RX J2135.9+4728 is clearly seen in this image near the location where the source IGR J21343+4738 was previously detected (Burenin et al. 2008). It lies at a distance of $\sim 15$ arcmin, which exceeds considerably both the IBIS localization accuracy and angular resolution. Based on other bright sources in the IBIS field of view, we can make sure that the astrometric errors in these observations are insignificant.

The sky field around IGR J21343+4738 was observed by the Chandra observatory on December 18 , 2006 (Sazonov et al. 2008). Based on the INTEGRAL observations, one may expect the source's brightness to drop significantly compared to that in the first observing period. However, owing to the high sensitivity of the Chandra observatory, a weak hard X-ray source was detected in the error region of the hard X-ray source IGR J21343+4738 that can be unambiguously associated with the optical object with the following coordinates $\alpha, \delta: 21^{\mathrm{h}} 34^{\mathrm{m}} 20 \leqq 37$ $+47^{\circ} 38^{\prime} 00^{\prime \prime}$. ( $\left.\mathrm{J} 2000\right)$. The finding chart for this field is shown in Fig. 6.

The spectrum of this object (Fig. 7) shows signatures of a B3 star. In particular, in addition to the overall shape of the spectrum, the fairly intense H I and $\mathrm{He}$ I absorption lines that are too strong for A type stars and and the absence of strong He II absorption lines typical of $\mathrm{O}$ stars point to the $\mathrm{B}$ spectral type. This implies that the $\mathrm{X}$-ray source is most likely a high-mass $X$-ray binary. In this case, the transient nature of its appearance in $X$ rays is not unusual, especially in view of the recent discovery of a large 
(a)

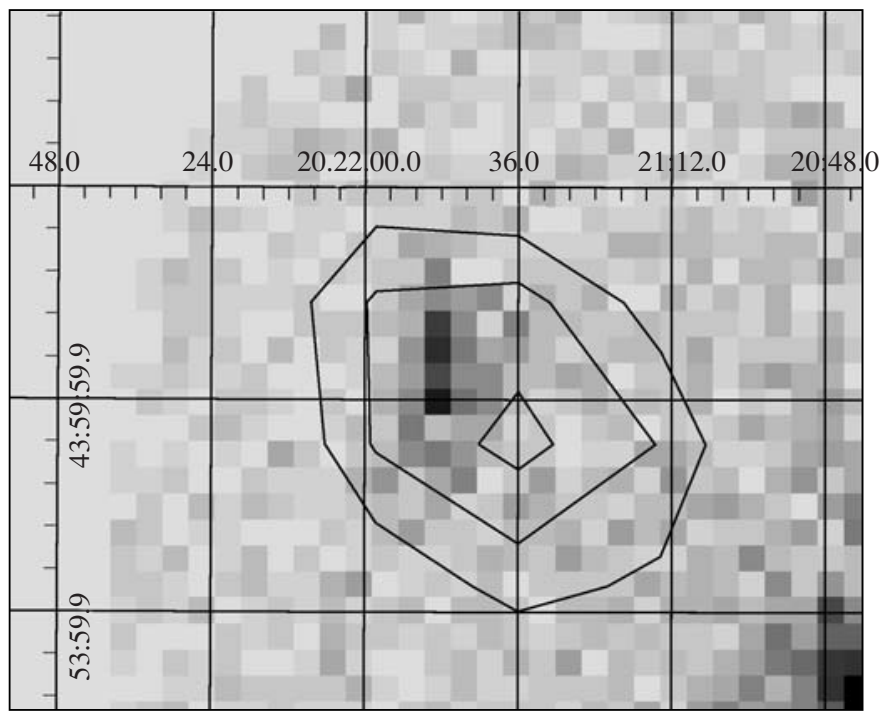

(b)

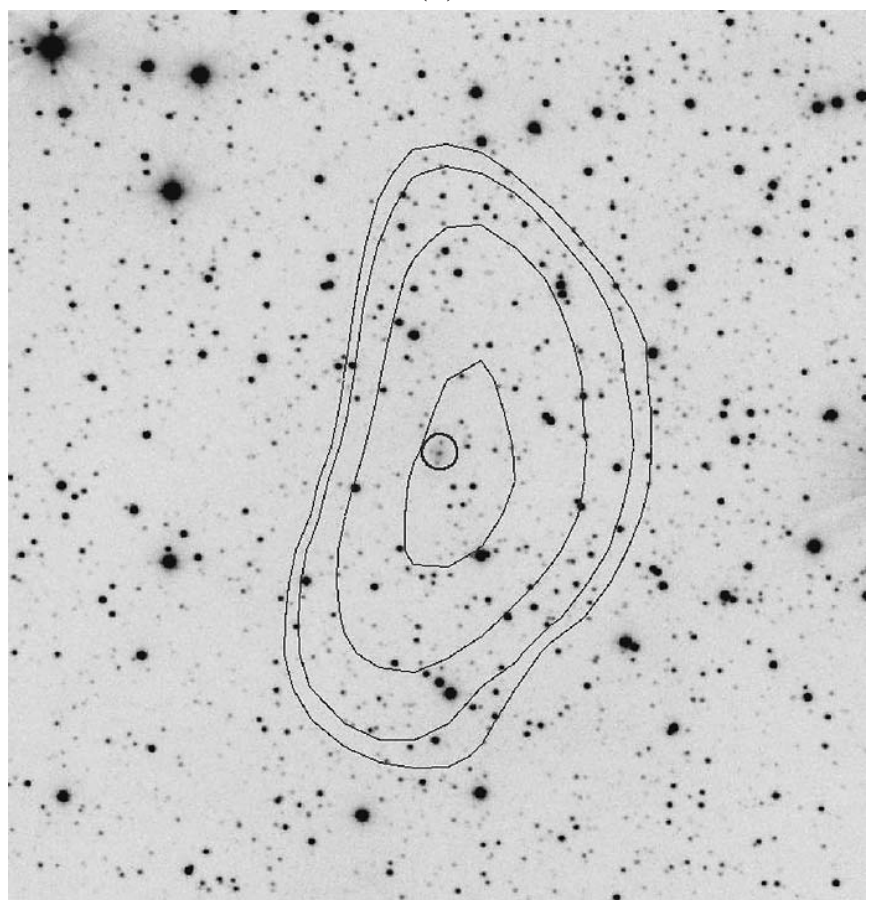

Fig. 3. (a) ASCA image of the sky field around the source IGR J20216+4359 in the energy range 4-10-keV obtained during the observations on June 10,1993. The contours indicate the regions where the IBIS/INTEGRAL fluxes exceeds the background value by 4.0, 4.5, and $5 \sigma$. (b) Optical image of the sky field around IGR J20216+4359 from RTT-150 observations. The contours indicate the ASCA position of the X-ray source. The circle marks the galaxy whose active nucleus is an X-ray source.

number of the so-called fast $X$-ray transients in highmass X-ray binaries (see, e.g., Chaty 2007).

The $\mathrm{H}_{\alpha}$ line in absorption, not in emission, as is usually observed in high-mass X-ray binaries, was detected in the objects's spectrum during the optical observations. This may be related to the long-period evolution of the equatorial disk wind from the optical companion similar to what is observed for some Besystems (see, e.g., Norton et al. 1991). The higherresolution $\approx 2 \AA$; Fig. $7 \mathrm{~b}$ ) echelle spectrum of the object taken with the TFOSC spectrometer showed an $\mathrm{H}_{\alpha}$ absorption line narrower than the He I $6678 \AA$ 


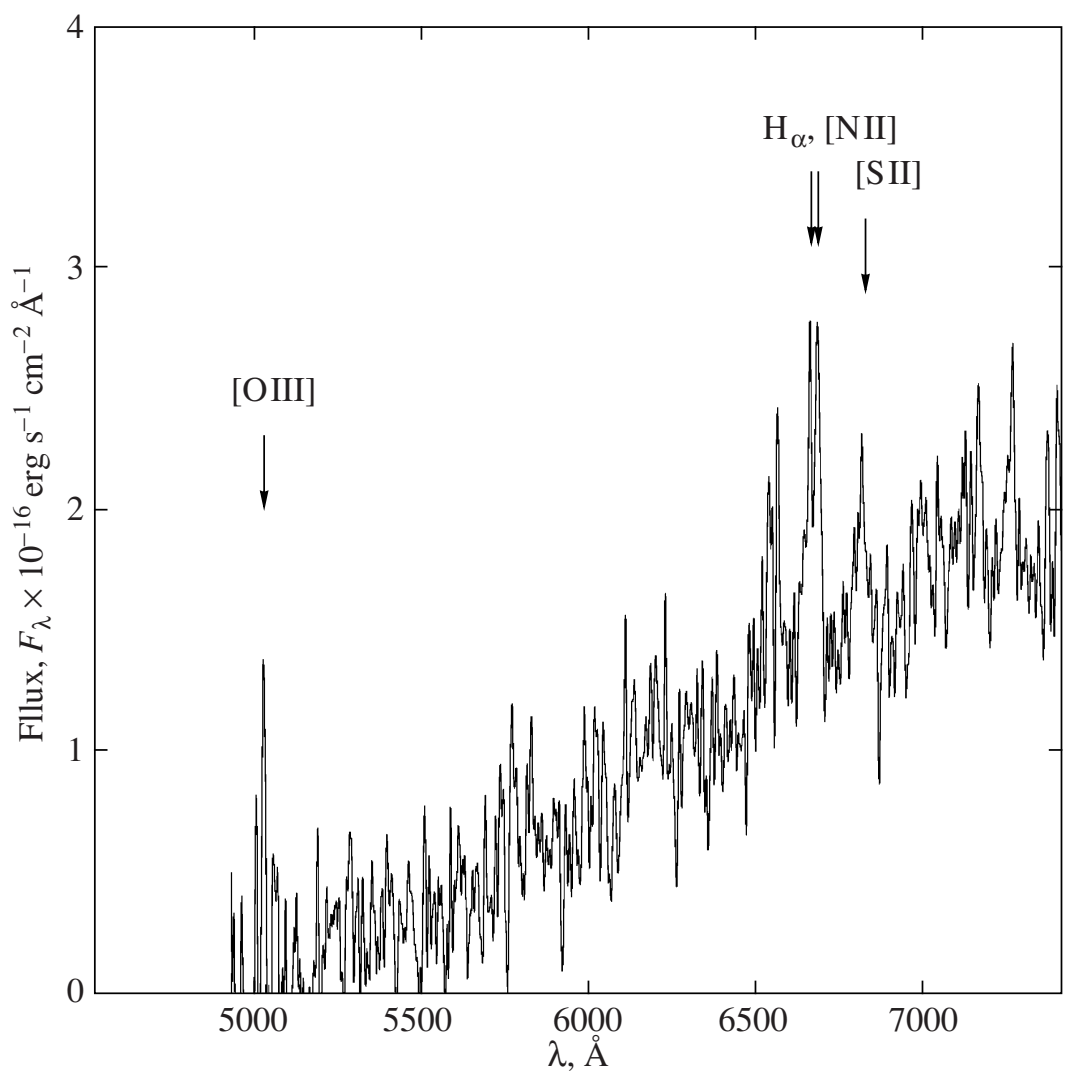

Fig. 4. RTT-150 spectrum of the source IGR J20216+4359 uncorrected for the Galactic extinction.

(a)

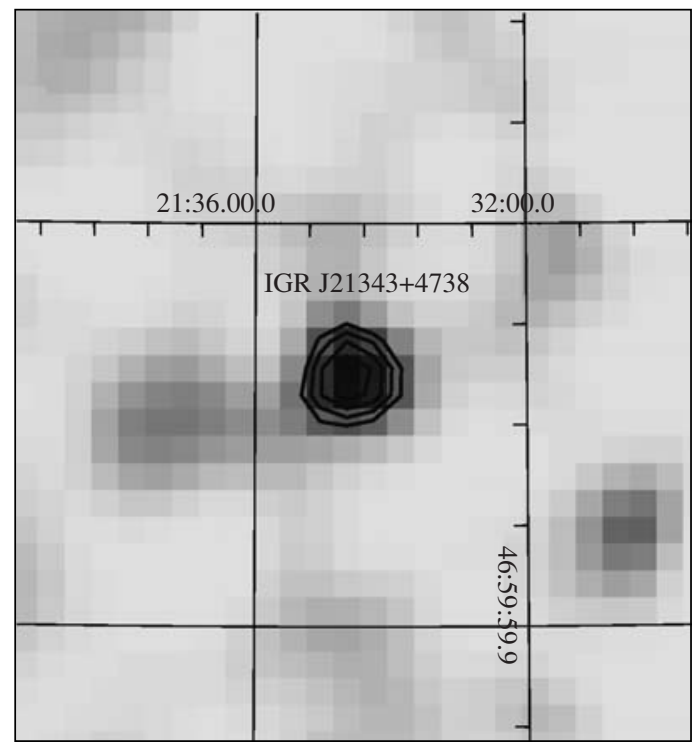

(b)

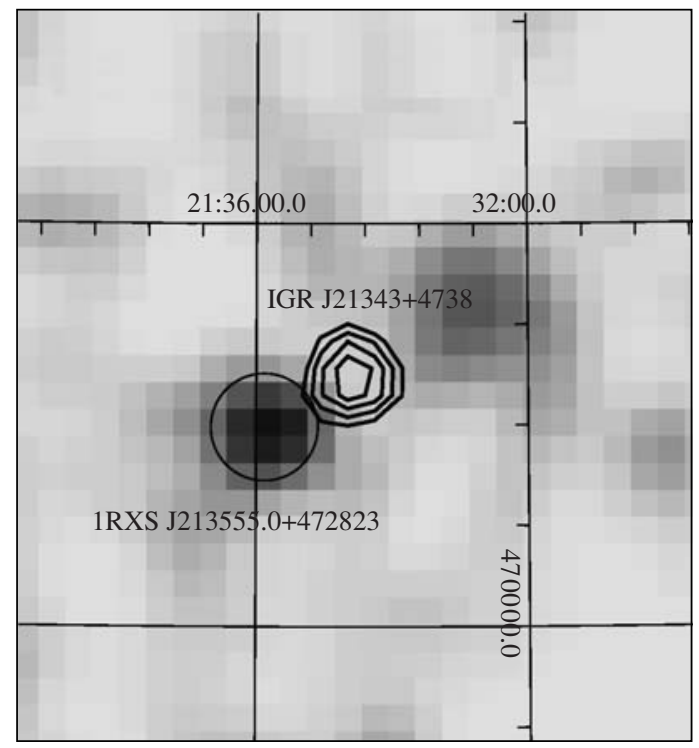

Fig. 5. INTEGRAL hard X-ray (17-60 keV) images of the sky field around IGR J21343+4738 (a) from December 27, 2002, to February 21, 2004, and (b) from March 11, 2004, to February 10, 2007. IGR J21343+4738 was clearly seen in the first observing period and was below the threshold in the second observing period. The AGN 1RXS J213555.0+472823 is seen near IGR J21343+4738 in the second observing period. The contours in the images bound the regions of equal statistical significance of the flux in the image obtained in the first observing period starting from $3.5 \sigma$ at $0.5 \sigma$ steps. 


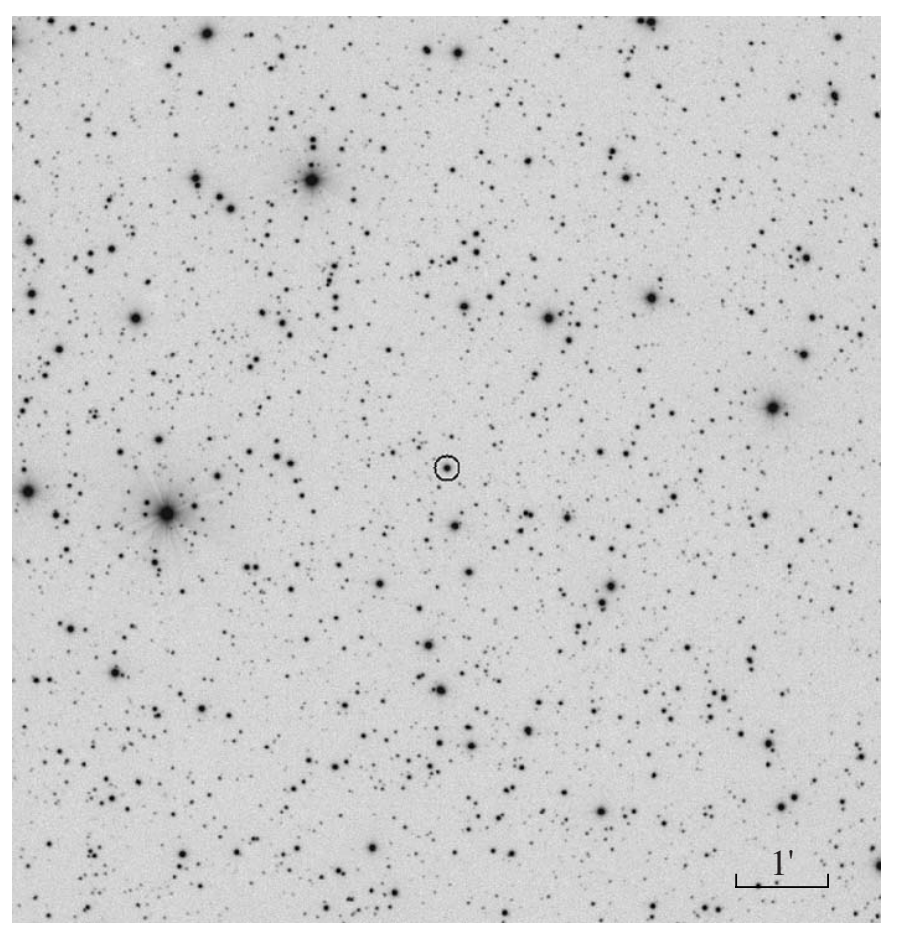

Fig. 6. Direct $R$-band image of the source IGR J21343+4738 obtained with the RTT-150 telescope.

line close to it, which may be indicative of a weak double-peaked emission from the equatorial disk of the optical star.

IGR J23206+6431. The hard X-ray source was discovered in the IBIS/INTEGRAL images of the Galactic plane already after the catalog of the allsky survey was published (Krivonos et al. 2007). The time-averaged $17-60-\mathrm{keV}$ flux from the source is $0.6 \pm 0.1 \mathrm{mCrab}$ or $\approx 8.7 \times 10^{-12} \mathrm{erg} \mathrm{s}^{-1} \mathrm{~cm}^{-2}$. This source was observed with the X-ray telescope onboard the SWIFT observatory, which allowed it to be unambiguously identified with the galaxy 2MASX J23203662+6430452.

Figure 8 shows the direct image of the source's field (a) and its spectrum (b) uncorrected for the Galactic extinction from RTT-150 observations. The optical spectrum of this object exhibits redshifted broad $\mathrm{H}_{\alpha}$ and narrow [O III] 5007 lines. Thus, this source is a type-1 Seyfert galaxy. Its redshift derived from the [O III] line is $z=0.07173$. Preliminary information about the optical identification of this source was immediately published in an astronomical circular (Bikmaev et al. 2008).

IGR J23523+5844. This hard X-ray source was observed by the Chandra observatory on January 14, 2007 (Sazonov et al. 2008). The X-ray absorption column density can be estimated from the Chandra X-ray spectrum to be $N_{\mathrm{H}} L=(3.7 \pm 0.5) \times$
$10^{22} \mathrm{~cm}^{-2}$, which is much higher than the absorption column density in our Galaxy (Dickey and Lockman 1990). This is a distinctive feature of the type 2 Seyfert galaxies in $\mathrm{X}$ rays.

An accurate position of the X-ray source allowed it to be identified with the optical object whose coordinates are given in the table. The finding chart for the field near this object is shown in Fig. 9a and its optical spectrum obtained with the SCORPIO spectrometer of the 6-m BTA telescope (Afanasiev and Moiseev, 2005) with a high-signal-to-noise ratio is presented in Fig. 9b. The spectrum of the optical object exhibits $\mathrm{Ca}, \mathrm{Mg}, \mathrm{Fe}$, and other absorption lines as well as intense narrow [O III]4959,5007, [S II]6717,6731, and probably [N II] 6548,6583 forbidden emission lines. Here, the flux ratio of the [O III] 5007 and $\mathrm{H}_{\beta}$ lines is definitely larger than ten, implying that the object can be identified as a Seyfert galaxy (see, e.g., Baldwin et al. 1981; Kauffmann et al. 2003). The absence of an intense broad $\mathrm{H}_{\beta}$ line suggests that this can be a type-2 Seyfert galaxy.

The redshift is $z=0.1620$; the $\mathrm{H}_{\alpha}$ and [N II]6548,6583 lines fall into the atmospheric $7600 \AA$ absorption band and their observations are complicated by the subtraction of a complex sky background. However, it can be said that the spectral feature that remains near the $7600 \AA$ band after the correction for the atmospheric absorption is 

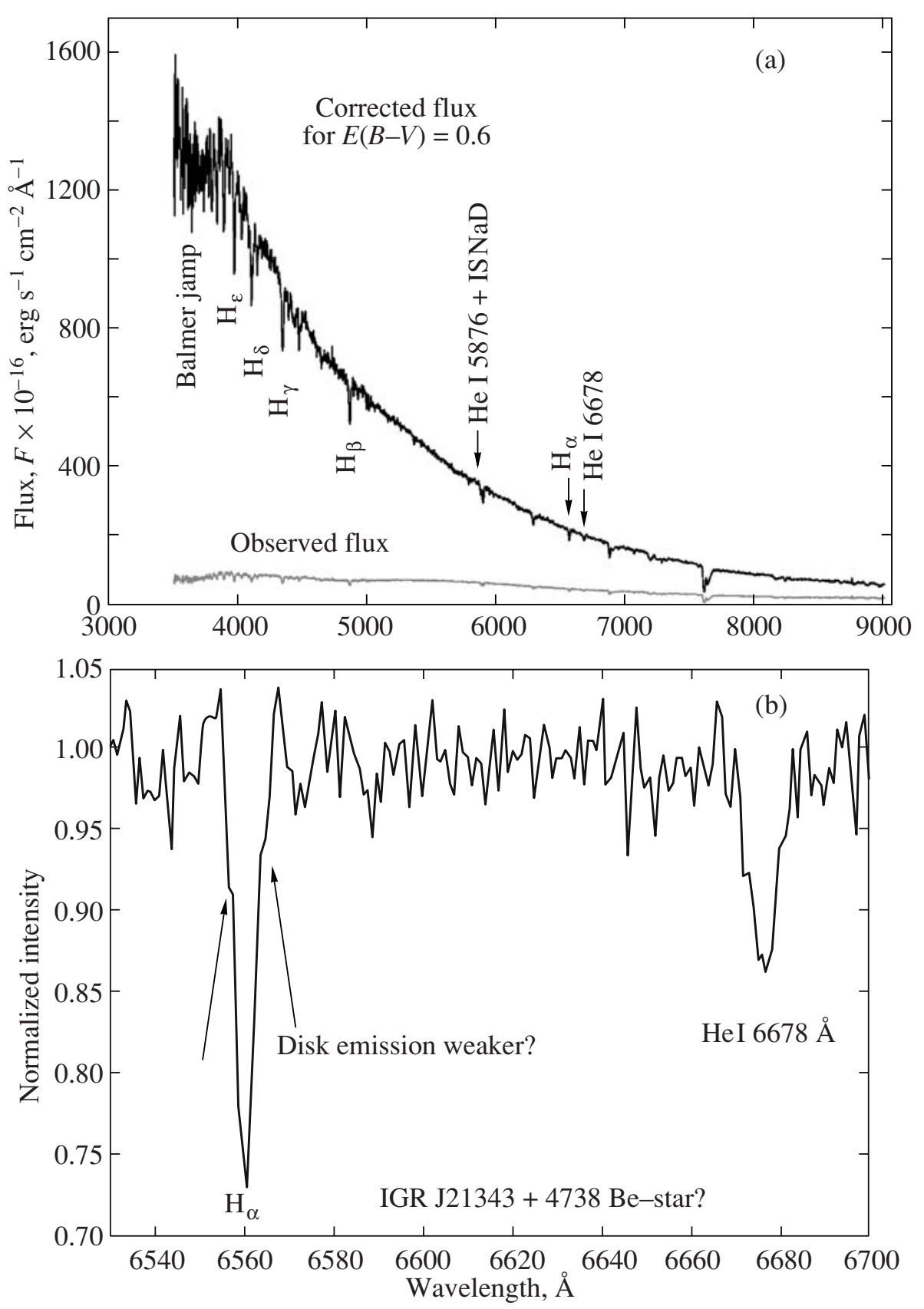

Fig. 7. (a) Spectrum of the optical object identified with the source IGR J21343+4738. (b) Higher-resolution spectrum of the source near the $\mathrm{H}_{\alpha}$ line. The arrows indicate the possible contribution from weak emission lines of the equatorial disk of a massive optical star.

redshifted from the $\mathrm{H}_{\alpha}$ line and, most likely, the [N II] 6583 forbidden line mainly contributes to the flux here. The spectrum also exhibits no traces of the narrow $\mathrm{H}_{\beta}$ and [O II] 3727 lines that are usually observed in the spectra of type-2 Seyfert galaxies. Thus, for example, the lower limit on the flux ratio of the [O III]5007 and $\mathrm{H}_{\beta}$ lines here is $\approx 20$, while the maximum value of this ratio for optically selected
AGNs is about 15 (see, e.g., Baldwin et al. 1981; Veilleux and Osterbrock 1987; Kauffmann et al. 2003). This may be because the regions in which the narrow hydrogen lines originate are strongly absorbed.

Note that the [O III] line emission in this AGN is spatially shifted from the continuum emission. This is clearly seen from the intensity distribution of the 
(a)

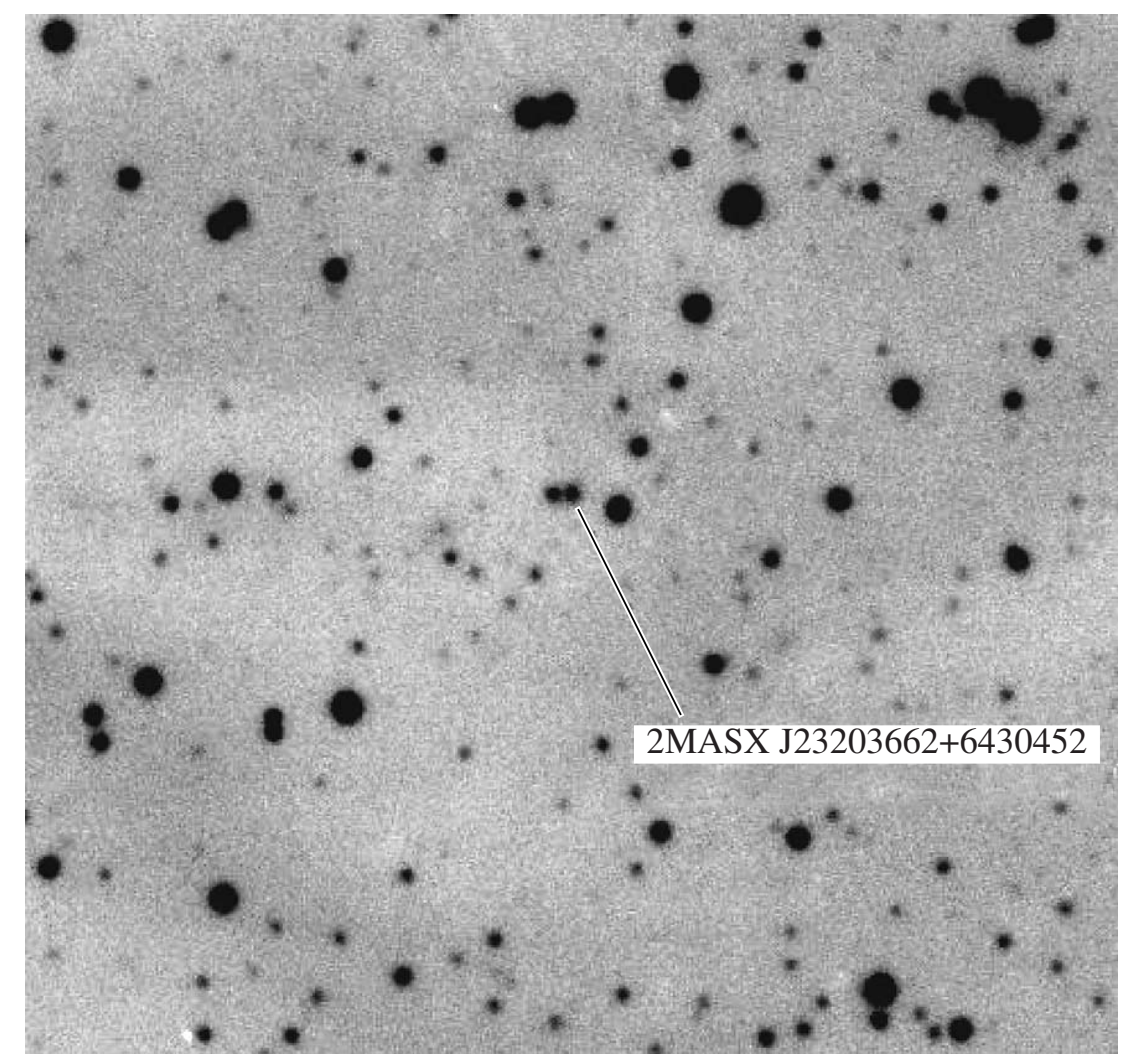

(b)

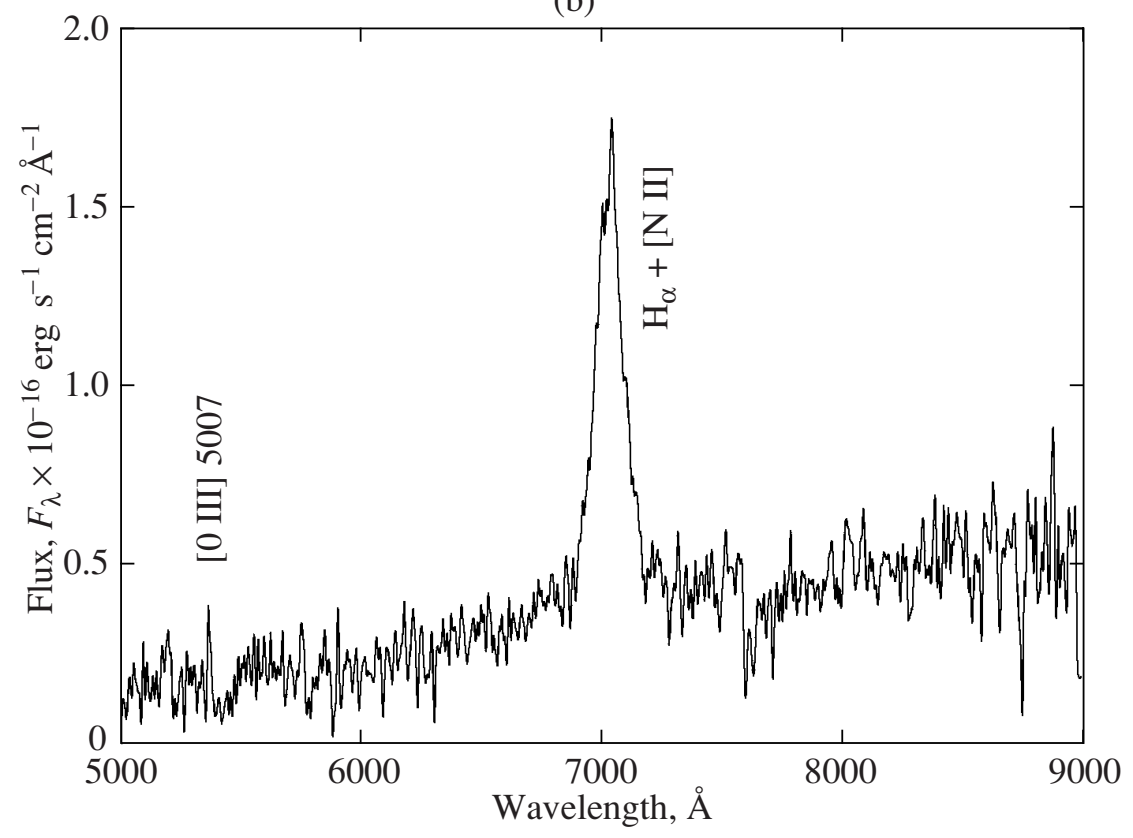

Fig. 8. (a) Optical $R$-band image of the IGR J23206+6431 field and (b) optical spectrum of the source from RTT-150 observations.

emission along the SCORPIO slit shown in Fig. 10. The difference in [O III] line and continuum surface brightness distributions is observed in some nearby
AGNs, for example, in Markarian 34 or Markarian 78, and reflects the fact that the emission in narrow forbidden lines originates at distances of $1-2 \mathrm{kpc}$ from 


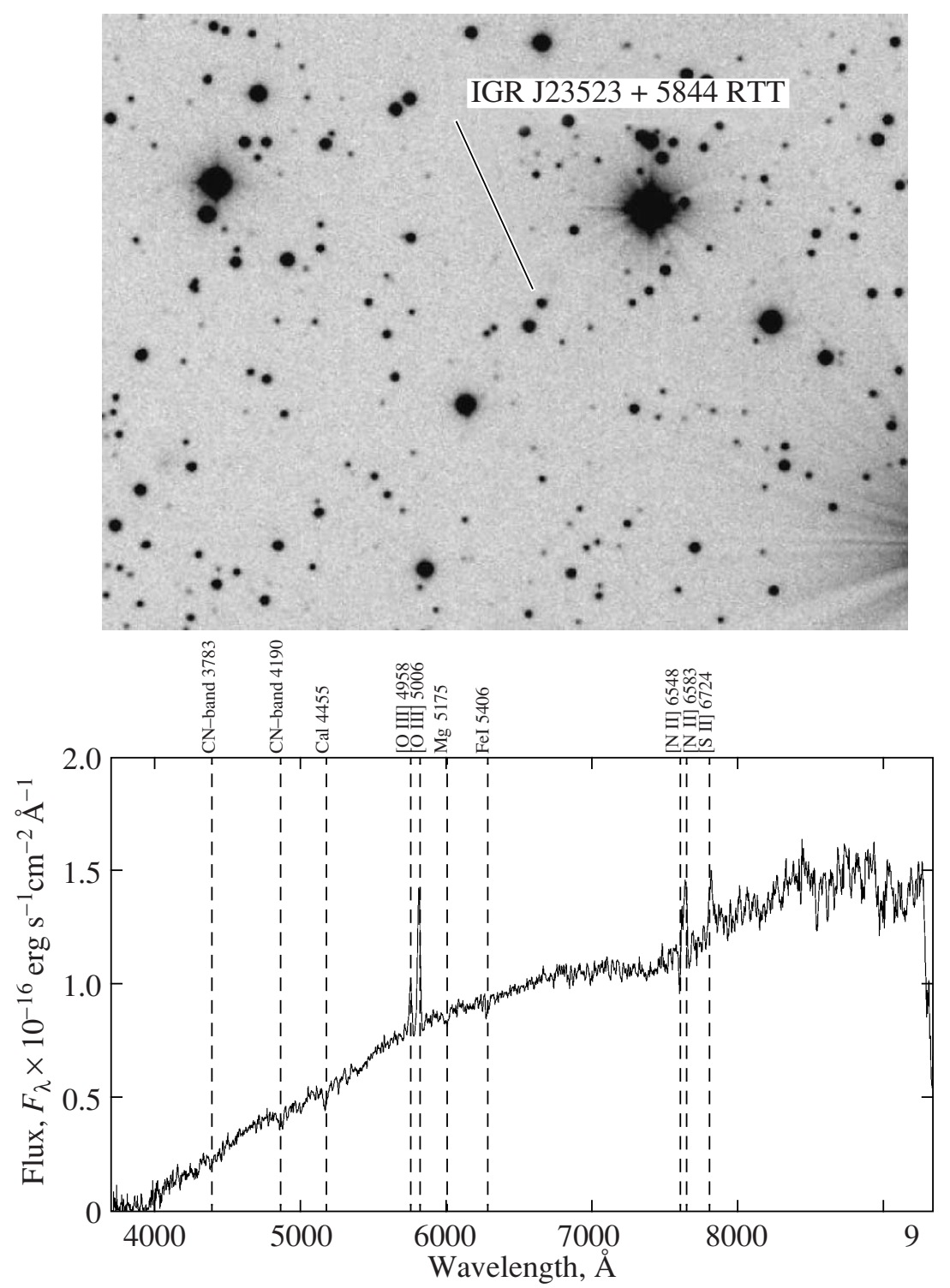

Fig. 9. (a) Optical image of the sky field around the source IGR J23523+5844 from RTT-150 observations. (b) Optical spectrum of the source from BTA observations uncorrected for the Galactic extinction.

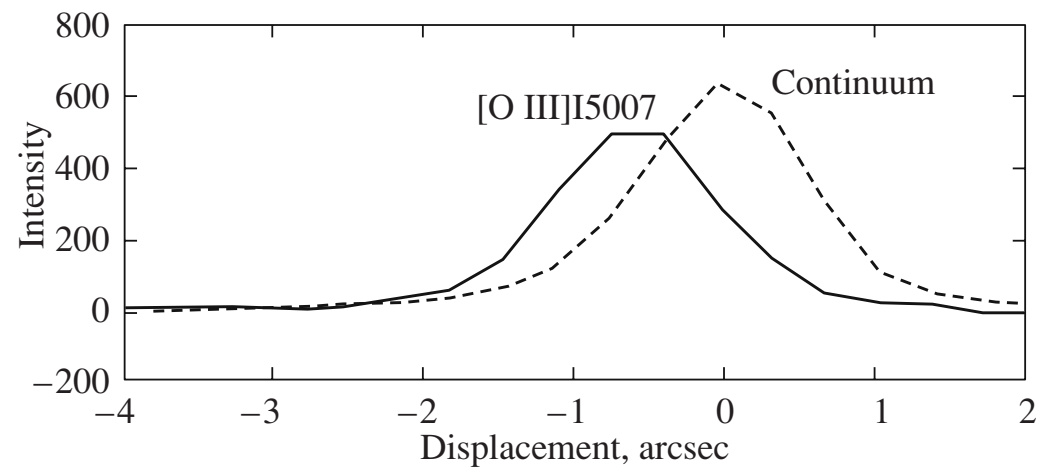

Fig. 10. Intensities of the continuum and [O III] line emissions from the AGN IGR J23523+5844 along the SCORPIO slit. 
the central black hole (see, e.g., Haniff et al. 1988). In addition, in the case of IGR J23523+5844, this agrees with the view that the central regions near the AGN are strongly absorbed and the observed emission in narrow forbidden lines originate only at considerable distances from the AGN.

Thus, all our data taken as a whole suggest that IGR J23523+5844 is most likely a type-2 Seyfert galaxy, although its optical spectrum may have some features related to strong absorption of the regions in which the narrow lines originate. In their paper recently published in preprints, Masetti et al. (2008) also identified this source as a probable type-2 Seyfert galaxy.

\section{CONCLUSIONS}

We presented information about the optical identifications of five hard X-ray sources from the INTEGRAL all-sky survey located near the Galactic plane. The X-ray data on one INTEGRAL source, IGR J20216+4359, are published for the first time. A blazar (RX J0137.7+5814), three Seyfert galaxies (IGR J20216+4359, IGR J23206+6431, and IGR J23523+5844), and a high-mass X-ray binary (IGR J21343+4738) are among the identified sources.

A peculiarity of this paper is that all of the identified objects are near the Galactic plane, i.e., in the region that is traditionally avoided by optical observers because of strong absorption and high star density. The INTEGRAL data can reveal new, hitherto unknown nearby AGNs that would be very difficult to find by their optical emission. As expected, there is also a large number of Galactic sources, mostly high-mass $\mathrm{X}$-ray binaries, among the hard $\mathrm{X}$-ray sources in the Galactic plane.

\section{ACKNOWLEDGMENTS}

We wish to thank V.L. Afanasiev for help with the optical observations at the 6-m BTA telescope and for a useful discussion of the observational results. We also wish to thank A.I. Galeev, R.Ya Zhuchkov (Kazan State University), and I.M. Khamitov (Turkish National Observatory) for help with the optical observations at the RTT-150 telescope. This work was supported by the Russian Foundation for Basic Research (project nos. 07-02-01004 and 07-0201051), the "Scientific School' Program (project nos. NSh-4224.2008.2 and NSh-1100.2006.2), and Programs P-04 and OFN-17 of the Russian Academy of Sciences.

\section{REFERENCES}

1. V. L. Afanasiev and A. V. Moiseev, Pis'ma Astron. Zh. 31, 214 (2005) [Astron. Lett. 31, 194 (2005)].

2. J. A. Baldwin, M. M. Phillips, and R. Terlevich, Publ. Astron. Soc. Pacific 93, 5 (1981).

3. I. F. Bikmaev, R. A. Sunyaev, M. G. Revnivtsev, and R. A. Burenin, Pis'ma Astron. Zh. 32, 250 (2006a) [Astron. Lett. 32, 221 (2006)].

4. I. F. Bikmaev, M. G. Revnivtsev, R. A. Burenin, and R. A. Sunaev, Pis'ma Astron. Zh. 32, 665 (2006b) [Astron. Lett. 32, 588 (2006)].

5. I. Bikmaev, M. Revnivtsev, R. Burenin, et al., Astron. Telegram. 1363 (2008).

6. A. J. Bird, A. Malizia, A. Bazzano, et al., Astrophys. J. Suppl. Ser. 170, 175 (2007).

7. R. A. Burenin, A. V. Meshcheryakov, M. G. Revnivtsev, et al., Pis'ma Astron. Zh. 34, 403 (2008) [Astron. Lett. 34, 367 (2008)].

8. S. Chaty, arXiv:0710.0292 (2007).

9. J. M. Dickey and F. J. Lockman, Ann. Rev. Astron. Astrophys. 28, 215 (1990).

10. G. Galazutdinov, DECH Software, Preprint SAO No. 92 (1992).

11. C. A. Haniff, A. S. Wilson, and M. J. Ward, Astrophys. J. 334, 104 (1988).

12. G. Kauffmann, T. M. Heckman, and C. Tremonti, Mon. Not. R. Astron. Soc. 346, 1055 (2003).

13. R. Krivonos, M. Revnivtsev, A. Lutovinov, et al., Astron. Astrophys. 475, 775 (2007).

14. A. Landolt, Astron. J. 104, 340 (1992).

15. N. Masetti, E. Mason, M. L. Morelli, et al., Astron. Astrophys., in press; arXiv:0802.0988 (2008).

16. D. G. Monet, S. E. Levine, B. Canzian, et al., Astrophys. J. 125, 984 (2003).

17. A. J. Norton, M. J. Coe, A. Estela, et al., Mon. Not. R. Astron. Soc. 253, 579 (1991).

18. S. Sazonov, E. Churazov, M. Revnivtsev, et al., Astron. Astrophys. 444, L37 (2005).

19. S. Sazonov, M. Revnivtsev, R. Burenin, et al., Astron. Astrophys., in press (2008); arXiv:0802.0928.

20. S. Veilleux and D. E. Osterbrock, Astrophys. J. Suppl. Ser. 63, 295 (1987).

21. W. Voges, B. Aschenbach, Th. Boller, et al., Astron. Astrophys. 349, 389 (1999).

Translated by A. Serber 